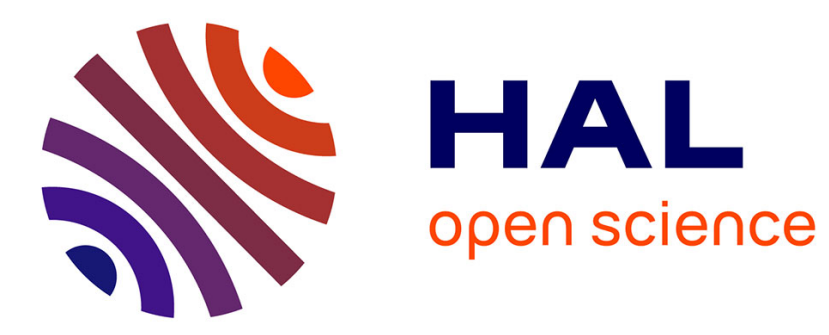

\title{
A New routing metric for satisfying both energy and delay constraints in wireless sensor networks
}

Najet Boughanmi, Ye-Qiong Song

\section{To cite this version:}

Najet Boughanmi, Ye-Qiong Song. A New routing metric for satisfying both energy and delay constraints in wireless sensor networks. 2nd International Conference on Sensor Networks and Applications (SNA06), Oct 2006, Beijing, China. inria-00105886

\section{HAL Id: inria-00105886 \\ https://hal.inria.fr/inria-00105886}

Submitted on 14 Nov 2006

HAL is a multi-disciplinary open access archive for the deposit and dissemination of scientific research documents, whether they are published or not. The documents may come from teaching and research institutions in France or abroad, or from public or private research centers.
L'archive ouverte pluridisciplinaire HAL, est destinée au dépôt et à la diffusion de documents scientifiques de niveau recherche, publiés ou non, émanant des établissements d'enseignement et de recherche français ou étrangers, des laboratoires publics ou privés. 


\title{
A NEW ROUTING METRIC FOR SATISFYING BOTH ENERGY AND DELAY CONSTRAINTS IN WIRELESS SENSOR NETWORKS
}

\author{
Najet Boughanmi and YeQiong Song \\ LORIA - INPL \\ Campus scientifique, BP239 \\ 54506 Vandoeuvre-lès-Nancy, France \\ emails : \{Najet.Boughanmi ; song\}@loria.fr
}

\begin{abstract}
Besides energy constraint, wireless sensor networks should also be able to provide bounded communication delay when they are used to support real-time applications. In this paper, a new routing metric is proposed. It takes into account both energy and delay constraints. By mathematical analysis and simulations, we have shown the efficiency of this new routing metric.
\end{abstract}

\section{INTRODUCTION}

With recent technical and technological advances in WSN (Wireless Sensor Network), it becomes now possible to envisage not only simple non real-time data collect but also more complicated real-time applications. Thus, WSN can be extended to include actuator nodes, called by some researchers wireless sensor and actuator network [1]. Each sensor node is composed of one or more sensors, a processor and a radio transmission unit. All of them are supplied by an unchangeable battery. Sensor nodes collect data from the environment that they are supervising and send them to other nodes or a base station (sink). This station processes received data and sends appropriate action commands to the actuators [2, 3]. Actuator nodes are assumed less energy constraint than the sensor nodes.

It is worth pointing out that the main research efforts in developing WSNs have focused on how to extend the network lifetime with respect to limited battery energy. However, when real-time applications are deployed on them, extending the lifetime of the network should be done without jeopardizing realtime communications from sensor nodes to other nodes or to data sinks. For example, a surveillance system needs to alert authorities of an intruder within a few seconds of detection [4]. Unfortunately, there is little work on the real-time communication support for WSNs.

For energy saving, most of work focuses on the communication protocol design since in a WSN the radio communication unit is the major power consumer in the node (it consumes about one thousand CPU units) [5]. IEEE 802.15.4 Task Group together with Zigbee Alliance [6] have developed an entire communication protocol stack for Low-Rate Personal Area Networks. One of the potential applications of this standard is in WSNs. This standard represents the new generation of distributed embedded systems for pervasive computing. IEEE 802.15.4 standard deals with the energy optimization in the physical layer and the Medium Access Control (MAC) sub-layer. Energy saving is mainly achieved by defining a sleeping period (inactive period) in a superframe. The Zigbee specifications define the routing and the application layer. The Zigbee routing protocol is almost the same as AODV with the exception of route maintenance. Even one may agree that AODV can always choose the route that minimizes the delay (or equivalently the number of hops), it does not take into account energy optimization. In this paper, we aim at improving the Zigbee routing protocol by including both energy and delay considerations.

Several energy-aware metrics have been proposed [7, $8,9]$ to optimize the energy consumption during the routing process. However they omit the real-time aspect. SPEED [10] deals with real-time applications. However it does not care about energy optimization in spite of its importance for the sensor network lifetime. [11] presents a routing approach which optimizes the network lifetime for real-time applications. However, it does not take into account the link's reliability. It should be noted that a route that chooses an unreliable link may experience longer delay because of the higher retransmission probability, which will in turn increase the energy consumption.

So, in this paper, we will focus on maximizing the sensor network lifetime while still taking into account the delay requirement of real-time communications. Our main idea is to find a new routing metric which is capable of including delay, energy, as well as link reliability factors. In our study, we used IEEE 802.15.4 protocol and Zigbee AODV. We are going to optimize the network lifetime under the delay constraint at the routing layer. Without loss of generality, the delay of a route is considered equivalent to the number of hops on the route and we assume that one can find the limit on the hop number for a given real-time communication constraint.

The rest of this paper is organized as follows. In Section 2, we outline related work. Section 3 provides a mathematical analysis for packet forwarding. We 
will give a routing metric that trades off between maximizing the sensor network lifetime and satisfying the communication delay. By simulations, we will compare the performance of our routing approach with the existing ones in Section 4. Finally, Section 5 gives conclusions and describes future directions.

\section{RELATED WORK}

Shortest hop counting is the most common metric used in both table-driven protocol such as DSDV (Destination-Sequenced Distance Victor) and sourceinitiated protocol such as AODV (Ad hoc On Demand Distance Victor) [12]. AODV is an on demand algorithm, which means that it builds routes between nodes only as desired by the sources. It maintains these routes as long as they are needed by the source. However, shortest-hop based routing is not suitable for wireless sensor networks since it neglects the energy issue.

Several routing protocols for wireless sensor networks have been concentrated on energy-aware issue because of its importance [7, 8, 9]. Cao et al. [9] propose an optimal bound on energy efficiency and try to achieve it asymptotically. Busse et al. [7] present two forwarding schemes named as SingleLink Energy-Efficient Forwarding and Multi-Link Energy-Efficient Forwarding respectively. These schemes maximize energy-efficiency and find a trade-off between delivery rate and energy cost. An enhancement of these schemes is presented in [8], which maximizes the lifetime efficiency by including the node's remaining energy into the forwarding metrics. These works do not guarantee the delay performance for real-time communications.

Speed [10] combines a real-time protocol and a location-based routing protocol such that each node chooses the next hop among its neighbor nodes, which are in "forwarding candidate set" of a message and satisfy the desired relay speed that refers to the delay constraint.

A lifetime efficient routing protocol for real-time applications in WSN is presented in [11]. Lifetime is maximized by choosing a set of energy efficient paths and transmitting alternatively over these routes to fairly balance energy consumption. Moreover, the delay constraint is embedded by ignoring routes that do not satisfy the maximum tolerable value. However, this routing approach does not consider link reliability. As previously mentioned, a route choosing an unreliable link may experience longer delay because of the higher retransmission probability, which in turn will increase the energy consumption.

\section{PROPOSED ROUTING METRIC}

\subsection{Model}

In this study, we adopt the model defined in [7]. This model captures the packet reception rate (PRR) between two nodes as follows. Nodes have full connectivity if they have a distance less than $D_{1}$. They are disconnected if they are separated by a distance greater than $D_{2}$. The expected PRR decreases smoothly in the transitive region between $D_{1}$ and $D_{2}$. The behavior is modeled by (1)

$$
P R R=\left\{\begin{array}{cc}
1 & \mathrm{~d}<\mathrm{D}_{1} \\
{\left[\frac{D_{2}-d}{D_{2}-D_{1}}+X\right]_{0}^{1}} & D_{1} \leq d \leq D_{2}{ }^{1} \\
0 & d>D_{2}
\end{array}\right.
$$

where $[.]_{a}^{b}=\max \{a, \min \{b,\}$.$\} and X \sim N\left(0, \sigma^{2}\right)$ is a Gaussian variable with variance $\sigma^{2}$.

\subsection{Metrics}

The wireless sensor network is presented by a graph $G=(V, A)$, in which $V$ is the set of nodes including the base station. The set of edges $A \subset V \times V$ such that $(i, j) \in A$ if nodes $i$ and $j$ can transmit to each other. To optimize the routing path, we assign each node the remaining energy and each vertex the delivery rate.

In the following, we are interested in the metric of the path efficiency. This metric considers the path energy efficiency and the delay experienced along this path. Here we are going to maximize energy efficiency while minimizing the delay together. Thus, we first define this path efficiency, $E$, to be the ratio of the path energy efficiency, $E_{\text {eff, }}$, to the delay required to transmit a packet from the source to the destination. The energy efficiency represents a trade-off between delivery rate and energy consumption along this path. In order to maximize the path efficiency and minimize the energy consumption, the energy efficiency is quantified as the ratio of the delivery rate, $E_{r}$, to the total energy consumed to transfer the packet to the destination node $E_{e}$. Thus, this energy efficiency is expressible by

$$
E_{e f f}=\frac{E_{r}}{E_{e}}
$$

The end-to-end delivery rate for a path $\varphi$ takes into account the delivery rate of each link in this path. So, this end-to-end delivery rate is the product of packet reception rate of each link in $\varphi$ as shown by

\footnotetext{
${ }^{1}$ This equation is modified, in numerator, $\mathrm{d}-\mathrm{D}_{1}$ is replaced by $D_{2}-d$ to find 1 when $d=D_{1}$.
} 


$$
E_{r}=\prod_{k \in \varphi, k \neq \text { destination }} \operatorname{prr}_{k, k+1}
$$

where $\operatorname{prr}_{k, k+1}$ is the packet reception rate between node $k$ and its forwarder $k+1$ as shown in Figure 1.

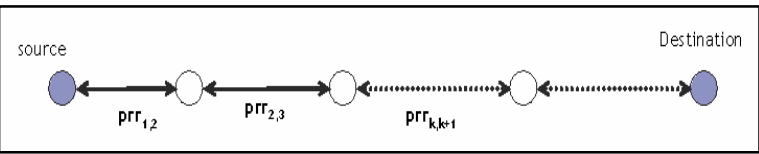

Fig. 1. Path

The required energy for the packet delivery is calculated by

$$
E_{e}=\operatorname{prr}_{i}\left(E_{e}^{i}+b\right)+a b
$$

where $p r_{i}$ is the packet reception rate for the forwarder $i, E_{e}{ }^{i}$ is its energy cost that refers to the energy consumption from the source to the node $i . b$ is the packet processing energy (transmission and reception) and $a=1-p r r_{i}$.

By replacing $E_{r}$ and $E_{e}$ in (2), the energy efficiency is given by

$$
E_{e f f}=\frac{\prod_{k \in \varphi, k \neq \text { destination }} \operatorname{prr}_{k, k+1}}{\operatorname{prr}_{i}\left(E_{e}^{i}+b\right)+a b}
$$

As the routing approach has to respect the delay to guarantee the "deadline" for real-time communications, the path efficiency can be calculated as

$$
E=\frac{\prod_{k \in \varphi, k \neq \text { destination }} \operatorname{prr}_{k, k+1}}{\text { delay } \cdot\left(\operatorname{prr}_{i}\left(E_{e}^{i}+b\right)+a b\right)}
$$

The routing approach presented by Coleri [11] guarantees the delay performance too. However, the corresponding delay is not included in the routing metric. In fact, in this approach only paths that offer delay less than the allowed delay are considered in the routing choice. Furthermore, the time is divided into time frames and at the beginning of each frame, the base station floods the network with a tree construction packet. Thus, there is significant energy consumption in the routing process. However, we use the AODV routing protocol with a modified routing metric as shown in (6). Hence, the route is setup according to the AODV request/response cycle. The delays are collected by route response message. Consequently, we have not increased the network load.

However, considering only the consumed energy is not sufficient to maximize the lifetime of the sensor network. We must include the remaining energy in the routing metric to balance the load of the network.
Thus the lifetime efficiency $E_{\mathrm{l}}$ is given by

$$
E_{l}=E_{\text {eff }} \cdot e_{i}
$$

where $e_{i}$ is the remaining energy of the forwarding node $i$.

By (5), the lifetime efficiency $E_{L}$ can be expressed as

$$
E_{l}=\frac{\prod_{k \in \varphi, k \neq \text { destination }} \operatorname{prr}_{k, k+1}}{\operatorname{prr}_{i}\left(E_{e}^{i}+b\right)+a b} \cdot e_{i}
$$

The new metric for the path efficiency which includes the lifetime efficiency can be calculated from

$$
E=\frac{\prod_{k \in \varphi, k \neq \text { destination }} \operatorname{prr}_{k, k+1}}{\text { delay } \cdot\left(\operatorname{prr}_{i}\left(E_{e}^{i}+b\right)+a b\right)} \cdot e_{i}
$$

Once we have defined our routing metric, we included it in the AODV routing protocol. Thus, our new version of AODV chooses the most efficient path to the destination node by considering both energy and delay constraints.

In Figure 2, we present an example of the execution of the routing process using our metric.

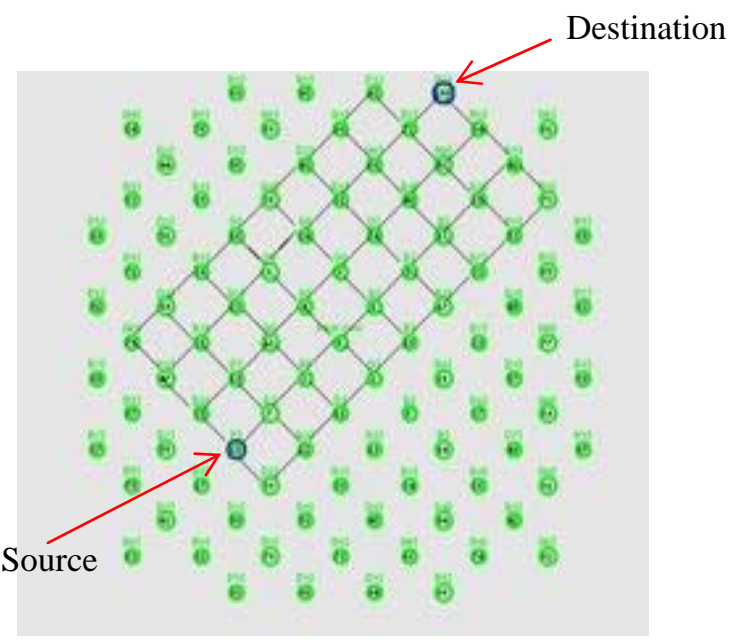

(A)

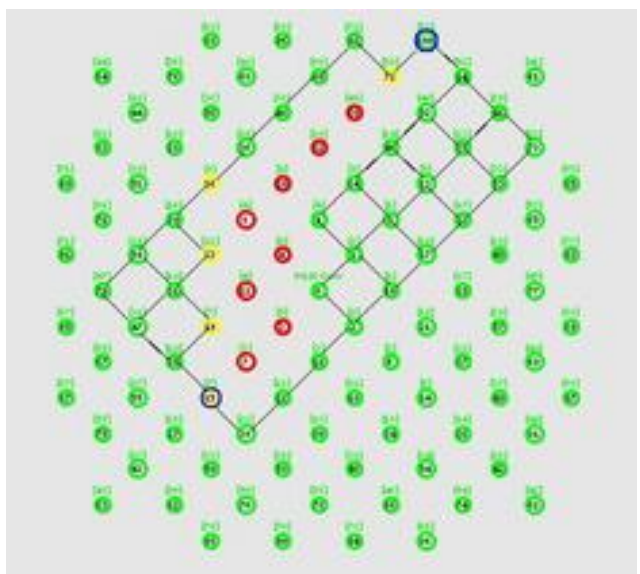

(B) 
Fig. 2 The source is at the lower left corner and the sink at the upper right corner of the graph. There are three energy levels presented by different colors. The green color is used for nodes with almost full energy. The yellow color is used for nodes with remaining energy less than threshold1. Finally red nodes have their remaining energy less than threshold2. (A) shows all possible paths from the source to the destination with hop's number $\leq 12$ at the beginning of the deployment. (B) shows the remaining paths after the death of some nodes (red nodes)

We can see that our metric chooses paths that satisfy our requirements.

\section{SIMULATION RESULTS AND DISCUSSION}

In this section, the performance of the proposed routing metric is evaluated and compared with AODV routing protocol and Coleri routing metric. Furthermore, we use NS-2 simulators to implement the physical and MAC layers of IEEE 802.15.4. We have changed the existing implementation in NS-2 of AODV to integrate our metric. Thus, we have a new version of AODV, which we call Enhanced AODV (E-AODV).

The primary purpose of our simulation is to observe the network lifetime resulted by our routing optimization. Moreover, we consider the delivery rate as another performance metric.

The simulated networks consist of 11, 22 and 101 nodes respectively.

\subsection{Assumptions}

The following assumptions are made in this study.

1. We consider a wireless sensor network that consists of a base station and several sensor nodes. These sensor nodes generate data for transfer to the base station. Delay constraint is only imposed on this sensor to base station communication.

2. Sensor nodes have a low mobility that is the case for most of the sensor network applications.

3. The delay needed to transmit a packet from a source node to a destination node is equivalent to the number of hops counted between these two nodes.

4. The operational lifetime of the sensor network is defined as the time until the first $5 \%$ of nodes died as proved in [11].

\subsection{Lifetime}

We study here the sensor network lifetime. We observe in Figure 3 and Figure 4 that at the beginning the three routing approaches have the same result. In fact, in the beginning of the network life, all nodes have a maximal amount of energy. Thus, the three routing approaches will have the same routing choices. Once the sensor energy decreases, the difference between these routing approaches appears. We observe in Figure 3 that the Enhanced AODV routing approach let sensors be alive for a longer time than AODV routing protocol does.

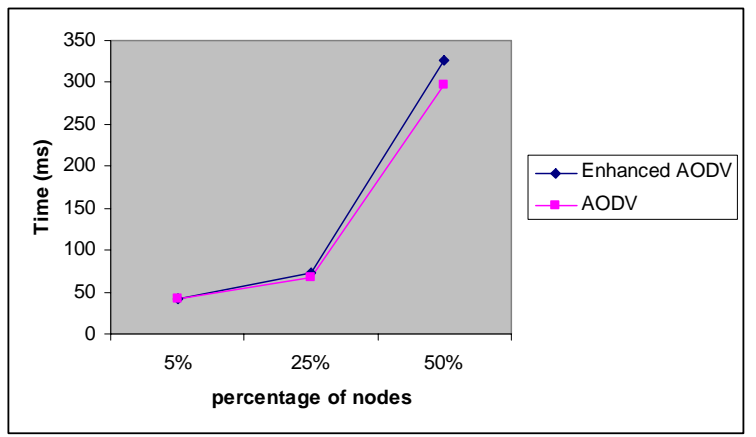

Fig. 3 Comparison of the time at which a specific percentage of the nodes are dead between Enhanced AODV and AODV.

Figure 4 shows that both of Enhanced AODV and Coleri routing metric give almost the same time for the death of a specific percentage of nodes. This is an expected result since both routing metrics aim to maximize sensor network lifetime.

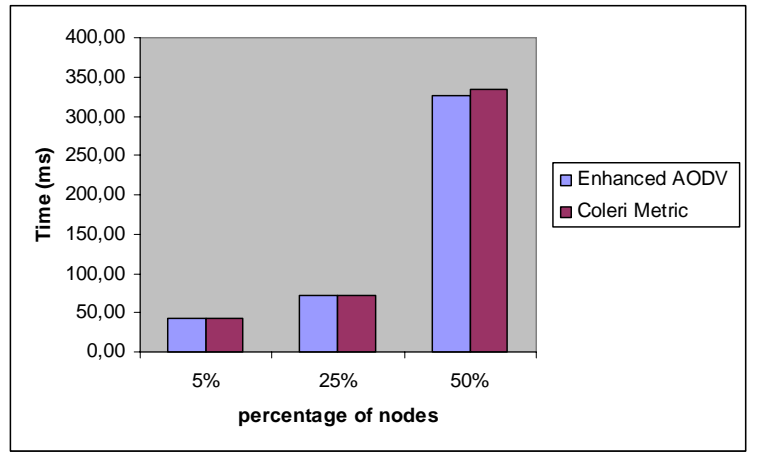

Fig. 4 Comparison of the time at which a specific percentage of the nodes are dead between Enhanced AODV and Coleri metric.

\subsection{Delivery rate}

In this sub-section, we focus on the optimization of the network delivery rate. We define the network delivery rate as the ratio of the total received packets to the total sent packets in the sensor network. We compute this delivery rate at different times in the sensor network lifetime and compare the results among Enhanced AODV, AODV and Coleri metric.

Figure 5 gives the delivery rate before the death of $5 \%$ of nodes. We notice that for a sensor network of a small number of nodes, all of the studied routing approaches offer the same delivery rate. In fact, in small sensor network there is almost one path from the source to destination. Thus, all of the routing algorithms choose the same path. However, for a network with a larger number of nodes, the Enhanced AODV performs better than AODV does. Moreover, the Enhanced AODV and Coleri routing metric gives almost the same delivery rate. 


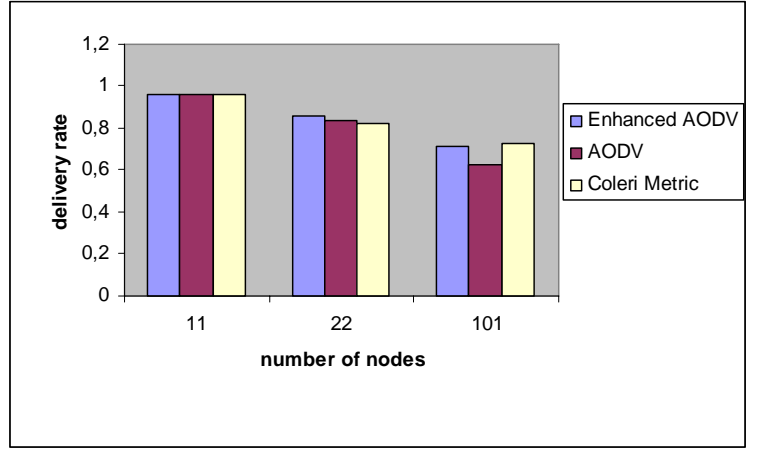

Fig. 5 Delivery rate before the death of $5 \%$ of nodes

Figure 6 shows the delivery rate before the death of $25 \%$ of nodes. In the same way as mentioned before, for a small sensor networks, all of the studied routing approaches give the same delivery rate. However, the benefit due to the optimization of delivery rate by the Enhanced AODV is clear. In fact, these routing approaches give better delivery rate than AOV and Coleri metric. Thus, although the Enhanced AODV and the Coleri metric offer the same network lifetime, the former gives a better delivery rate.

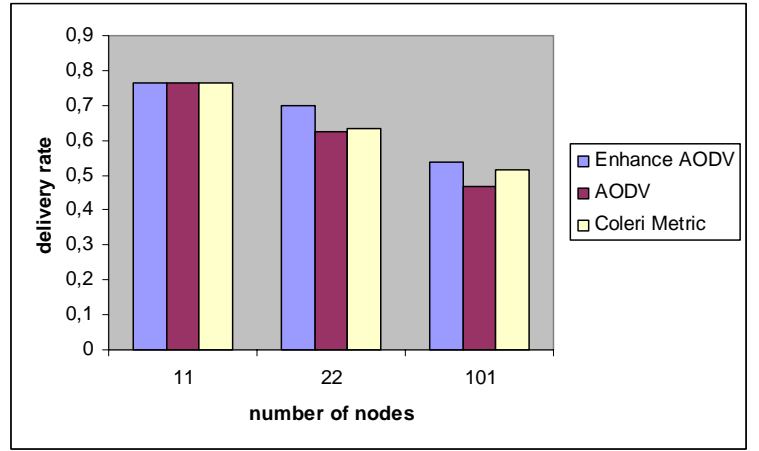

Fig. 6 Delivery rate before the death of $25 \%$ of nodes

From the results given by the Figure 7 we notice that the Enhanced AODV offers better delivery rate than AODV and Coleri routing approaches. Thus, for different moment of the network lifetime, the delivery rate is always better with the Enhanced AODV routing approach.

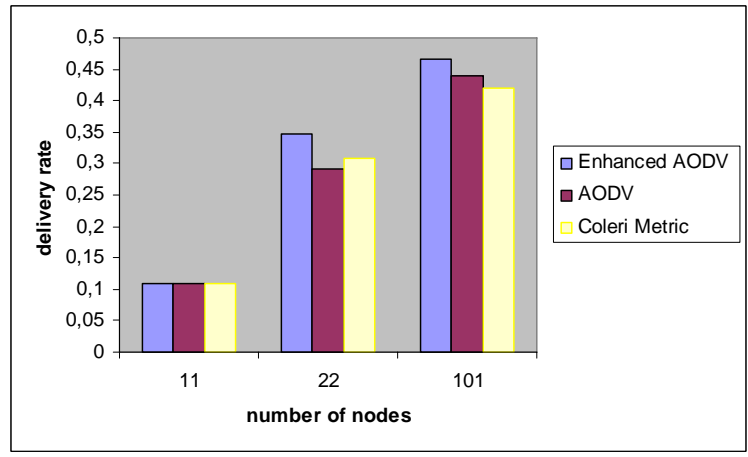

Fig. 7 Delivery rate before the death of $50 \%$ of nodes

\section{CONCLUSION}

A successful deployment of real-time applications over WSNs needs to satisfy the required timing properties under energy consumption constraints. As existing solutions do not address energy and delay issues together at the same time, we propose in this paper a new routing metric. The benefit of this metric has been shown by simulations when embedded into AODV protocol. The same metric may also be used in other routing protocols. For instance, this metric can be used in data centric routing [13] if we include packet's importance in it. Therefore we can classify packets into two levels according to there relevance: the normal level and the urgent level. The packets with the normal level are not allowed to go through nodes with a critical energy level. However, the urgent packets can be routed in paths containing nodes with a critical energy level.

The communication reliability is another important criterion for real-time application deployment. This issue must be addressed since sensor nodes may not be reliable because of their low cost and the hostile environment in which they are deployed. Some works dealing with the reliability can be found, for example ReInForM [14] which calculates the number of parallel forwarding nodes needed at each hop to achieve a specified end-to-end success probability. However, ReInForM does not scale well with the number of sinks. Indeed, each sink needs a separate cost at every node. Thus, the amount of state increases proportionally to the number of sinks. Moreover, it does not optimize the energy consumption. By using our developed simulation model, we will go to evaluate the probability of the real-time communication guarantee during the network lifetime in our future work. In addition we will compare the reliability of our routing approach to that given by ReInForM .

\section{REFERENCES}

[1] I. F. Akyildiz and I. H. Kasimoglu, "Wireless sensor and actor networks: research challenges", Ad hoc networks, 2 (2004), pp 351-367, May 2004.

[2] Y. Li, Z. Wang, and Y.Q. Song, "Wireless sensor network design for wildfire monitoring", $6^{\text {th }}$ World Congress on Intelligent Control and Automation (WCICA 2006), Dalian (China), 2006.

[3] C. Lu, G. Xing, O. Chipara, C.L. Fok, and S. Bhattacharya, "A spatiotemporal query service for mobile users in sensor networks", ICDCS, pp. 381390, 2005.

[4] T. He, P. A. Vicaire, T. Yan, L. Luo, L. Gu, G. Zhou, R. Stoleru, Q. Cao, J. A. Stankovic and T. Abdelzaher, "Achieving real-time target tracking using wireless sensor networks ", $12^{\text {th }}$ IEEE Real-Time and Embedded Technology and Applications Symposium, pp. 37-48, April 2006.

[5] S. Coleri Ergen, and P. Varaiya, "PEDAMACS: Power 
efficient and delay aware medium access protocol for sensor networks", IEEE Transactions on Mobile Computing, vol. 5, pp. 920-930, July 2006.

[6] Zigbee Specifications, 2004. http://www.zigbee.org.

[7] M. Busse, T. Haenselmann, and W. Effelsberg, "An Energy-Efficient Forwarding Scheme for Wireless Sensor Networks”, Technical report 13, University of Mannheim, Dec. 2005.

[8] M. Busse, T. Haenselmann, and W. Effelsberg, "PosterAbstract: A Lifetime-Efficient Forwarding Strategy for Wireless Sensor Networks”, EWSN, 2006.

[9] Q. Cao, T. He, L. Fang, T. Abdelzaher, J. Stankovic, and S. Son, "Efficiency Centric Communication Model for Wireless Sensor Networks", Infocom, 2006.

[10] T. He, J. A Stankovic, C. Lu, and T. Abdelzaher, "SPEED: A Stateless Protocol for Real-Time Communication in Sensor Networks", Proceedings of the International Conference on Distributed Computing Systems (IDCS 2003), May 2003.

[11] S. Coleri Ergen, and P. Varaiya, "Energy Efficient Routing with Delay Guarantee for Sensor Networks", ACM Wireless Networks WINET, 2006.

[12] E. M. Royer, and C. E. Perkins, "An Implementation Study of the AODV Routing Protocol”, Proceedings of the IEEE Wireless Communications and Networking Conference, Chicago, IL, September 2000.

[13] C. Intanagonwiwat, R. Govindan, and D. Estrin, "Directed Diffusion: A Scalable and Robust Communication Paradigm for Sensor Networks", Proc. of ACM MOBICOM , 2000.

[14] B. Deb, S. Bhatnagar, and B. Nath, "ReInForM: Reliable Information Forwarding Using Multiple Paths in Sensor Networks", in 28th Annual IEEE Conference on Local Computer Networks, 2003. 